\title{
Assessment of Different Microbial Population in the Rhizosphere of Main Kharif Crop under Conventional and Conservation Agriculture System
}

\author{
Kritika Dongre*, B. Sachidanand and S.S. Porte \\ Department of Soil Science and Agriculture Chemistry, Jawaharlal Nehru Agriculture \\ University, Jabalpur (M.P.), India \\ *Corresponding author
}

\section{A B S T R A C T}

Keywords

Conventional

tillage,

Conservational

tillage, Zero tillage,

Microbial

population.

Article Info

Accepted:

14 July 2017

Available Online:

10 September 2017
Conventional tillage practices may adversely affect long-term soil productivity due to erosion and loss of organic matter in soils. Sustainable soil management can be practiced through conservation tillage (including no-tillage), high crop residue return, and crop rotation. Microorganisms in the soil strongly influence soil processes fulfill key roles in the decomposition of organic matter, Therefore, the constituents of soil microorganisms, such as microbial and microbial community diversity, have often been identified as sensitive indicators of biological indices for maintaining soil health and quality. Conservational agriculture system showed significantly higher total bacterial count in rhizospheric soil of main kharif crop viz. paddy, maize and soybean over conventional agriculture system. Conservational agriculture system proved to be superior over conventional agriculture system. Microbial activity as bacteria, actinomycetes fungi were highest at reproductive stage in both conventional and conservational agriculture system in soybean crop.

\section{Introduction}

Microorganisms in the soil strongly influence soil processes (Garbeva et al., 2004), fulfil key roles in the decomposition of organic matter, the cycling of carbon and nitrogen and the formation and stabilization of soil structure (Loranger-Merciris et al., 2006).

Therefore, the constituents of soil microorganisms, such as microbial and microbial community diversity, have often been identified as sensitive indicators of biological indices for maintaining soil health and quality (Bending et al., 2004). Unsustainable agricultural practices have resulted in extreme soil erosion (Cai et al., 2006; Jiang et al., 2007), which can lead to physical and chemical degradation (Lal et al., 2000). In response to the decline in regional soil quality in the Loess plateau, there has been a gradual shift from conventional tillage towards conservation tillage practices such as zero tillage farming, crop residue retention and crop rotation. These production practices, have resulted in positive effects on crop yield and soil physical and chemical properties (Peixoto et al., 2006), also strongly influence the size, composition, diversity and function of soil microbial communities (Steenwerth et 
al.,2002; Salles et al., 2006), resulting in significantly altered soil processes. Zero tillage has increased soil organic carbon in the surface layer (Melero et al., 2009), including increases of up to $100 \%$ in microbial biomass $\mathrm{C}$ (MB-C) in as little as five years (Franchini et al., 2007), and significantly improved soil microbial activity and diversity as well. Conservation tillage is defined as a tillage system in which at least $30 \%$ of crop residues are left in the field and is an important conservation practice to reduce soil erosion. The advantages of conservation tillage practices over conventional tillage include reducing cultivation cost; allowing crop residues to act as an insulator and reducing soil temperature fluctuation; building up soil organic matter; conserving soil moisture. Conventional tillage can lead to soil microbial communities dominated by aerobic microorganisms, while conservation tillage practices increase microbial population and activity as well as microbial biomass.

\section{Materials and Methods}

Two field sites were selected for the study in the research field of Department of Soil Science and Agricultural Chemistry, JNKVV Jabalpur under AICRP on STCR and The Bourlog Institute of South Asia situated at village Khamaria Jabalpur. The initial fertility status of soil before sowing of each crop was tested. Composite soil samples from $0-15 \mathrm{~cm}$ and $15-30 \mathrm{~cm}$ depth was randomly collected from the experimental field. All the possible technical precautions as prescribed for standard soil sampling method have been followed. The soil samples thus obtained from two depth $0-15 \mathrm{~cm}$ and $15-30 \mathrm{~cm}$ were subjected to various chemical and biological analysis to assess the two field sites for analysis of soil biochemical properties and microbial population counts before sowing of the crop maximum vegetative growth and after harvest of each crop under conventional and conservational agriculture system. The experimental data were collected subjected to statistical analysis to test the significance of treatments and statistical analysis was done following method using Factorial CRD.

\section{Results and Discussion}

The findings obtained during the investigation are discussed with possible reasons for superiority of the system supported with the findings by research workers in India and abroad on various characters under study. Attempts have been made in the following description to evaluate and explain the effect of different treatments on various parameters of paddy, maize and soybean crop recorded in the investigation. The data collected for each parameter was compiled, statistically analyzed and being presented in tables 1-6.

It is evident from the data that paddy crop $\left(\mathrm{C}_{1}\right)$ at initial stage $\left(\mathrm{St}_{1}\right)$ under conservational agriculture system the bacterial count was 14.57 percent higher than conventional agriculture system. At reproductive stage in both the system $\left(S_{1}\right.$ and $\left.S_{2}\right)$, the bacterial count was low which may be due to submerged condition. At harvest stage the bacterial count was 36.25 percent higher in conservational agriculture system in paddy rhizosphere soil. In maize crop $\left(\mathrm{C}_{2}\right)$ at initial stage $\left(\mathrm{St}_{1}\right)$ bacterial count was 20.30 percent recorded at reproductive stage $\left(\mathrm{St}_{2}\right)$ it was 2.72 percent increase and at harvest 45.25 percent higher was recorded in conservational agriculture system. In soybean crop $\left(\mathrm{C}_{3}\right)$ at initial stage $\left(\mathrm{St}_{1}\right)$ bacterial count was 62.42 percent, while at reproductive stage it was 13.4 percent higher than conventional agriculture system. Bacterial count was significantly high in surface soil $0-15 \mathrm{~cm}$ depth than 15-30 cm depth of soil in both the system $\left(\mathrm{S}_{1}\right)$ conventional and $\left(\mathrm{S}_{2}\right)$ conservational respectively. The interaction studies between crop stages, crop depth, and crop and system has been presented in table 2 was observed that all the interaction studied 
were significant. The interaction of crop with stage of growth was observed to be maximum microbial count at initial stage $\left(\mathrm{St}_{1}\right)$ with paddy (33.12) and soybean was (31.54) while it was $49.75 \mathrm{cfu} / \mathrm{g}$ being maximum at harvest $\left(\mathrm{St}_{3}\right)$ in case of maize. Interaction effect with depth and crop indicated significant microbial count of all the crops in $0-15 \mathrm{~cm}$ depth having $28.4,44.03$, and $48.71 \mathrm{cfu} / \mathrm{g}$ of bacteria in paddy, maize and soybean respectively. The interaction of system of cultivation with crop has been also found to be differing significantly over conventional in case of maize and soybean having 51.99 and 46.69 respectively, while paddy crop had highest population count at conventional system (22.30) while conservational system had
(21.95) cfu/g.

It is observed that the microbial population at all the stages was higher in the conservational system than conventional system.

It is evident from data that paddy crop $\left(\mathrm{C}_{1}\right)$ at initial stage $\left(\mathrm{St}_{1}\right)$ under conservational agriculture system the actinomycetes count was 2.14 percent higher than conventional agriculture system. At reproductive stage in both the system $\left(\mathrm{S}_{1}\right.$ and $\left.\mathrm{S}_{2}\right)$ the actinomycetes count was low which was be attributed due to submerged condition and at harvest the actinomycetes count was 8.51 percent higher in conservational agriculture system.

Table.1 Impact of conventional and conservational agriculture system in bacterial count $(\mathrm{cfu} / \mathrm{g})$ of rhizospheric soil $\left(10^{5}\right)$

\begin{tabular}{|c|c|c|c|c|}
\hline Depth & Crop & Stage & $\begin{array}{l}\text { S1 } \\
\text { Conventional } \\
\left(10^{5}\right)\end{array}$ & $\begin{array}{l}\text { S2 } \\
\text { Conservational } \\
\left(\mathbf{1 0}^{5}\right)\end{array}$ \\
\hline \multirow{9}{*}{$\begin{array}{l}0-15 \mathrm{~cm} \\
\left(\mathrm{D}_{1}\right)\end{array}$} & \multirow{3}{*}{$\operatorname{Paddy}\left(\mathrm{C}_{1}\right)$} & $\operatorname{Initial}\left(\mathrm{St}_{1}\right)$ & 63.45 & 72.70 \\
\hline & & Reproductive $\left(\mathrm{St}_{2}\right)$ & 10.78 & 10.84 \\
\hline & & Harvest $\left(\mathrm{St}_{3}\right)$ & 10.45 & 14.24 \\
\hline & \multirow{3}{*}{$\operatorname{Maize}\left(\mathrm{C}_{2}\right)$} & $\operatorname{Initial}\left(\mathrm{St}_{1}\right)$ & 61.32 & 73.77 \\
\hline & & Reproductive $\left(\mathrm{St}_{2}\right)$ & 13.97 & 14.35 \\
\hline & & Harvest $\left(\mathrm{St}_{3}\right)$ & 12.55 & 18.23 \\
\hline & \multirow{3}{*}{$\operatorname{Soybean}\left(\mathrm{C}_{3}\right)$} & $\operatorname{Initial}\left(\mathrm{St}_{1}\right)$ & 32.63 & 53.00 \\
\hline & & Reproductive $\left(\mathrm{St}_{2}\right)$ & 31.69 & 74.35 \\
\hline & & Harvest $\left(\mathrm{St}_{3}\right)$ & 37.47 & 63.10 \\
\hline \multirow{9}{*}{$\begin{array}{l}15-30 \mathrm{~cm} \\
\left(\mathrm{D}_{2}\right)\end{array}$} & \multirow{3}{*}{$\operatorname{Paddy}\left(\mathrm{C}_{1}\right)$} & $\operatorname{Initial}\left(\mathrm{St}_{1}\right)$ & 29.52 & 33.07 \\
\hline & & Reproductive $\left(\mathrm{St}_{2}\right)$ & 8.73 & 9.50 \\
\hline & & Harvest $\left(\mathrm{St}_{3}\right)$ & 10.85 & 3.34 \\
\hline & \multirow{3}{*}{$\operatorname{Maize}\left(C_{2}\right)$} & $\operatorname{Initial}\left(\mathrm{St}_{1}\right)$ & 27.76 & 31.60 \\
\hline & & Reproductive $\left(\mathrm{St}_{2}\right)$ & 2.65 & 4.84 \\
\hline & & Harvest $\left(\mathrm{St}_{3}\right)$ & 28.14 & 28.59 \\
\hline & \multirow{3}{*}{$\operatorname{Soybean}\left(\mathrm{C}_{3}\right)$} & $\operatorname{Initial}\left(\mathrm{St}_{1}\right)$ & 28.39 & 34.23 \\
\hline & & Reproductive $\left(\mathrm{St}_{2}\right)$ & 22.66 & 49.14 \\
\hline & & Harvest $\left(\mathrm{St}_{3}\right)$ & 26.29 & 45.32 \\
\hline SEm \pm & \multicolumn{4}{|l|}{0.621} \\
\hline $\mathrm{CD}(\mathrm{p}=0.05)$ & \multicolumn{4}{|l|}{1.86} \\
\hline
\end{tabular}


Table.2 Interactions between crop (C) and stage (St), depth (D) and crop, system (S) and crop (C) on bacterial population in $(\mathrm{cfu} / \mathrm{g})$

\begin{tabular}{|l|l|l|l|l|l|l|l|}
\hline \multicolumn{2}{|l}{ Crop x Stages } & \multicolumn{2}{l|}{ Crop x Depth } & \multicolumn{2}{l|}{ Crop x System } \\
\hline Crop & $\begin{array}{l}\text { Initial } \\
\left(\mathbf{S t}_{\mathbf{1}}\right)\end{array}$ & $\begin{array}{l}\text { Reproductive } \\
\left.\mathbf{( S t}_{\mathbf{2}}\right)\end{array}$ & $\begin{array}{l}\text { Harvest } \\
\left.\mathbf{( S t}_{\mathbf{3}}\right)\end{array}$ & $\begin{array}{l}\mathbf{0 - 1 5 c m} \\
\left(\mathbf{D}_{\mathbf{1}}\right)\end{array}$ & $\begin{array}{l}\mathbf{1 5 - 3 0 c m} \\
\left(\mathbf{D}_{\mathbf{2}}\right)\end{array}$ & Conv. & Cons. \\
\hline Paddy $\left(\mathbf{C}_{1}\right)$ & 33.12 & 6.3 & 4.8 & 28.4 & 15.84 & 22.30 & 21.95 \\
\hline Maize $\left(\mathbf{C}_{2}\right)$ & 32.4 & 4.3 & 49.75 & 44.03 & 42.43 & 34.47 & 51.99 \\
\hline Soybean $\left(\mathbf{C}_{3}\right)$ & 31.54 & 22.8 & 28.69 & 48.71 & 34.34 & 36.36 & 46.69 \\
\hline Mean & 32.36 & 11.14 & 27.75 & 40.38 & 30.87 & 31.04 & 40.21 \\
\hline SEm \pm & & & $\mathbf{0 . 3 1}$ & & $\mathbf{0 . 2 5}$ & & $\mathbf{0 . 2 5}$ \\
\hline CD $(\mathbf{p = 0 . 0 5 )}$ & & & $\mathbf{0 . 9 3}$ & & $\mathbf{0 . 7 5}$ & & $\mathbf{0 . 7 5}$ \\
\hline
\end{tabular}

Table.3 Impact of conventional and conservational agricultural system on actinomycetes count (cfu/g) of rhizospheric soil $\left(10^{5}\right)$

\begin{tabular}{|c|c|c|c|c|}
\hline Depth & Crop & Stage & $\begin{array}{l}\text { S1 } \\
\text { Conventional } \\
\left(10^{5}\right)\end{array}$ & $\begin{array}{l}\text { S2 } \\
\text { Conservational } \\
\left(\mathbf{1 0}^{5}\right)\end{array}$ \\
\hline \multirow{9}{*}{$\begin{array}{l}0-15 \mathrm{~cm} \\
\left(\mathrm{D}_{1}\right)\end{array}$} & \multirow{3}{*}{$\operatorname{Paddy}\left(\mathrm{C}_{1}\right)$} & $\operatorname{Initial}\left(\mathrm{St}_{1}\right)$ & 16.28 & 16.63 \\
\hline & & Reproductive $\left(\mathrm{St}_{2}\right)$ & 0.35 & 0.36 \\
\hline & & Harvest $\left(\mathrm{St}_{3}\right)$ & 12.21 & 13.25 \\
\hline & \multirow{3}{*}{$\operatorname{Maize}\left(\mathrm{C}_{2}\right)$} & $\operatorname{Initial}\left(\mathrm{St}_{1}\right)$ & 16.29 & 16.31 \\
\hline & & Reproductive $\left(\mathrm{St}_{2}\right)$ & 28.71 & 64.63 \\
\hline & & Harvest $\left(\mathrm{St}_{3}\right)$ & 24.33 & 24.89 \\
\hline & \multirow[t]{3}{*}{ Soybean $\left(C_{3}\right)$} & $\operatorname{Initial}\left(\mathrm{St}_{1}\right)$ & 17.03 & 17.29 \\
\hline & & Reproductive $\left(\mathrm{St}_{2}\right)$ & 8.13 & 8.20 \\
\hline & & Harvest $\left(\mathrm{St}_{3}\right)$ & 6.25 & 5.01 \\
\hline \multirow{9}{*}{$\begin{array}{l}15-30 \mathrm{~cm} \\
\left(D_{2}\right)\end{array}$} & \multirow[t]{3}{*}{$\operatorname{Paddy}\left(\mathrm{C}_{1}\right)$} & $\operatorname{Initial}\left(\mathrm{St}_{1}\right)$ & 16.23 & 28.28 \\
\hline & & Reproductive $\left(\mathrm{St}_{2}\right)$ & 0.40 & 0.27 \\
\hline & & Harvest $\left(\mathrm{St}_{3}\right)$ & 26.62 & 33.14 \\
\hline & \multirow[t]{3}{*}{$\operatorname{Maize}\left(C_{2}\right)$} & $\operatorname{Initial}\left(\mathrm{St}_{1}\right)$ & 16.51 & 26.66 \\
\hline & & Reproductive $\left(\mathrm{St}_{2}\right)$ & 28.69 & 79.85 \\
\hline & & Harvest $\left(\mathrm{St}_{3}\right)$ & 28.80 & 53.38 \\
\hline & \multirow[t]{3}{*}{ Soybean $\left(C_{3}\right)$} & Initial $\left(\mathrm{St}_{1}\right)$ & 16.24 & 26.40 \\
\hline & & Reproductive $\left(\mathrm{St}_{2}\right)$ & 8.03 & 10.28 \\
\hline & & Harvest $\left(\mathrm{St}_{3}\right)$ & 5.41 & 5.05 \\
\hline SEm \pm & \multicolumn{4}{|l|}{0.53} \\
\hline$C D(p=0.05)$ & \multicolumn{4}{|l|}{1.59} \\
\hline
\end{tabular}


Table.4 Interaction between crop(c) and stage(St), depth (D) and crop (C), system(s) and crop(c) on actinomycetes population (cfu/g)

\begin{tabular}{|l|l|l|l|l|l|l|l|}
\hline \multicolumn{3}{|l}{ Crop x Stage } & \multicolumn{2}{l|}{ Depth x Crop } & \multicolumn{2}{l|}{ System x Crop } \\
\hline Crop & $\begin{array}{l}\text { Initial } \\
\left(\mathbf{S t}_{\mathbf{1}}\right)\end{array}$ & $\begin{array}{l}\text { Reproductive } \\
\left(\mathbf{S t}_{\mathbf{2}}\right)\end{array}$ & $\begin{array}{l}\text { Harvest } \\
\left(\mathbf{S t}_{\mathbf{3}}\right)\end{array}$ & $\begin{array}{l}\mathbf{0 -} \\
\mathbf{1 5 c m} \\
\left(\mathbf{D}_{\mathbf{1}}\right)\end{array}$ & $\begin{array}{l}\mathbf{1 5 - 3 0 c m} \\
\left(\mathbf{D}_{\mathbf{2}}\right)\end{array}$ & Conv. & Cons. \\
\hline Paddy $\left(\mathrm{C}_{1}\right)$ & 12.9 & 0.2 & 14.2 & 9.85 & 17.49 & 15.34 & 11.99 \\
\hline Maize $\left(\mathrm{C}_{2}\right)$ & 12.63 & 33.58 & 20.90 & 29.19 & 37.91 & 44.19 & 22.91 \\
\hline Soybean $\left(\mathrm{C}_{3}\right)$ & 12.83 & 5.77 & 3.62 & 10.32 & 11.90 & 11.88 & 10.34 \\
\hline Mean & 12.7 & 13.19 & 12.91 & 16.45 & 22.43 & 23.80 & 15.08 \\
\hline SEm \pm & & & $\mathbf{0 . 2 6}$ & & $\mathbf{0 . 2 1}$ & & $\mathbf{0 . 2 1}$ \\
\hline $\mathbf{C D}(\mathbf{p}=\mathbf{0 . 0 5})$ & & & $\mathbf{0 . 7 8 5}$ & & $\mathbf{0 . 6 3 1}$ & & $\mathbf{0 . 6 3 1}$ \\
\hline
\end{tabular}

Table.5 Impact of conventional and conservational agricultural system in fungal count (cfu/g) of rhizospheric soil $\left(10^{5}\right)$

\begin{tabular}{|c|c|c|c|c|}
\hline Depth & Crop & Stage & $\begin{array}{l}\text { S1 } \\
\text { Conventional } \\
\left(10^{5}\right) \\
\end{array}$ & $\begin{array}{l}\text { S2 } \\
\text { Conservational } \\
\left(10^{5}\right) \\
\end{array}$ \\
\hline \multirow{9}{*}{$\begin{array}{l}0-15 \mathrm{~cm} \\
\left(\mathrm{D}_{1}\right)\end{array}$} & \multirow{3}{*}{$\operatorname{Paddy}\left(\mathrm{C}_{1}\right)$} & $\operatorname{Initial}\left(\mathrm{St}_{1}\right)$ & 34.50 & 35.92 \\
\hline & & Reproductive $\left(\mathrm{St}_{2}\right)$ & 73.87 & 92.00 \\
\hline & & Harvest $\left(\mathrm{St}_{3}\right)$ & 7.26 & 17.17 \\
\hline & \multirow{3}{*}{$\operatorname{Maize}\left(\mathrm{C}_{2}\right)$} & $\operatorname{Initial}\left(\mathrm{St}_{1}\right)$ & 31.41 & 35.53 \\
\hline & & Reproductive $\left(\mathrm{St}_{2}\right)$ & 18.64 & 20.27 \\
\hline & & Harvest $\left(\mathrm{St}_{3}\right)$ & 25.41 & 27.43 \\
\hline & \multirow{3}{*}{ Soybean $\left(\mathrm{C}_{3}\right)$} & $\operatorname{Initial}\left(\mathrm{St}_{1}\right)$ & 32.64 & 32.67 \\
\hline & & Reproductive $\left(\mathrm{St}_{2}\right)$ & 13.40 & 29.00 \\
\hline & & Harvest $\left(\mathrm{St}_{3}\right)$ & 6.38 & 20.20 \\
\hline \multirow{9}{*}{$\begin{array}{l}15-30 \mathrm{~cm} \\
\left(\mathrm{D}_{2}\right)\end{array}$} & \multirow{3}{*}{$\operatorname{Paddy}\left(\mathrm{C}_{1}\right)$} & $\operatorname{Initial}\left(\mathrm{St}_{1}\right)$ & 26.62 & 34.17 \\
\hline & & Reproductive( $\left.\mathrm{St}_{2}\right)$ & 12.06 & 11.79 \\
\hline & & Harvest $\left(\mathrm{St}_{3}\right)$ & 2.96 & 4.59 \\
\hline & \multirow[t]{3}{*}{ Maize $\left(\mathrm{C}_{2}\right)$} & $\operatorname{Initial}\left(\mathrm{St}_{1}\right)$ & 25.33 & 22.60 \\
\hline & & Reproductive $\left(\mathrm{St}_{2}\right)$ & 7.87 & 11.97 \\
\hline & & Harvest $\left(\mathrm{St}_{3}\right)$ & 16.59 & 19.17 \\
\hline & \multirow[t]{3}{*}{ Soybean $\left(\mathrm{C}_{3}\right)$} & $\operatorname{Initial}\left(\mathrm{St}_{1}\right)$ & 24.82 & 25.90 \\
\hline & & Reproductive $\left(\mathrm{St}_{2}\right)$ & 7.92 & 11.07 \\
\hline & & Harvest $\left(\mathrm{St}_{3}\right)$ & 6.61 & 8.25 \\
\hline SEm \pm & \multicolumn{4}{|l|}{\begin{tabular}{|l|l|}
0.87 \\
\end{tabular}} \\
\hline $\mathrm{CD}(\mathrm{p}=0.05)$ & \multicolumn{4}{|l|}{2.61} \\
\hline
\end{tabular}


Table.6 Interaction between crop (C), stages (St), depth (D), crop and system(S) and crop on fungi population in (cfu/g)

\begin{tabular}{|c|c|c|c|c|c|c|c|}
\hline \multicolumn{4}{|l|}{ Crop x Stage } & \multicolumn{2}{|c|}{ Depth x Crop } & \multicolumn{2}{|c|}{ System x Crop } \\
\hline Crop & $\begin{array}{l}\text { Initial } \\
\left(\mathbf{S t}_{1}\right)\end{array}$ & $\begin{array}{l}\text { Reproductive } \\
\left(\mathbf{S t}_{2}\right)\end{array}$ & $\begin{array}{l}\text { Harvest } \\
\left(\mathbf{S t}_{\mathbf{3}}\right)\end{array}$ & $\begin{array}{l}0-15 \mathrm{~cm} \\
\left(D_{1}\right)\end{array}$ & $\begin{array}{l}15-30 \mathrm{~cm} \\
\left(D_{2}\right)\end{array}$ & Conv. & Cons. \\
\hline $\operatorname{Paddy}\left(\mathrm{C}_{1}\right)$ & 21.9 & 31.62 & 5.33 & 43.45 & 15.37 & 29.38 & 29.44 \\
\hline $\operatorname{Maize}\left(\mathrm{C}_{2}\right)$ & 19.15 & 9.79 & 14.77 & 26.45 & 17.25 & 21.88 & 21.83 \\
\hline Soybean $\left(\mathrm{C}_{3}\right)$ & 19.34 & 10.23 & 6.91 & 22.38 & 14.10 & 15.46 & 21.01 \\
\hline Mean & 20.12 & 17.21 & 9.00 & 30.76 & 15.57 & 22.24 & 24.09 \\
\hline SEm \pm & & & 0.43 & & 0.35 & & 0.35 \\
\hline $\mathrm{CD}(\mathrm{p}=0.05)$ & & & 1.29 & & 1.05 & & 1.05 \\
\hline
\end{tabular}

In maize crop $\left(\mathrm{C}_{2}\right)$ at initial stage $\left(\mathrm{St}_{1}\right)$ actinomycetes count was 1.5 percent at reproductive stage $\left(\mathrm{St}_{2}\right) 12.7$ percent and at harvest 2.30 percent higher in conservational agriculture system. In soybean crop $\left(\mathrm{C}_{3}\right)$ at initial stage $\left(\mathrm{St}_{1}\right)$ actinomycetes count was 1.52 percent more, and at reproductive stage it was 3.4 percent higher than conventional agriculture system.

Actinomycetes count was high in sub surface soil $\left(D_{2}\right) 15-30 \mathrm{~cm}$ than $\left(D_{1}\right) 0-15 \mathrm{~cm}$ in both the system $\left(\mathrm{S}_{1}\right)$ conventional and $\left(\mathrm{S}_{2}\right)$ conservational respectively in all the crops.

The data presented in table 4 shows the interaction between crop, depth, stage and system effect on the actinomycetes population count. The interaction between crop and stages showed that in paddy crop the actinomycetes count was 12.9 which abruptly decreased to 0.2 almost nil and then increased to 14.2 at harvest $\left(\mathrm{St}_{3}\right)$. The maize crop also had similar count at initial stage $\left(\mathrm{St}_{1}\right) 12.63$ and increased to 33.58 in reproductive stage $\left(\mathrm{St}_{2}\right)$ and 20.90 at harvest $\left(\mathrm{St}_{3}\right)$ being highest of crop.

In case of soybean the count was almost similar to paddy and maize which gradually decreased at reproductive stage $\left(\mathrm{St}_{2}\right)$ and at harvest $\left(\mathrm{St}_{3}\right)$ stages having $12.83,5.77$ and 12.9 in initial stage respectively. When compared with the depth and crop it is observed that at $0-15 \mathrm{~cm}$ depth had 9.85, 29.19 and 10.32 actinomycetes count was recorded which was significantly lower than the count at second depth $(15-30 \mathrm{~cm})$ having $17.49,37.91$ and 11.90 at initial, reproductive and harvest stage in crop paddy, maize and soybean respectively.

The interaction of system with crop, was recorded that maize crop had more population in both the system having 44.19 and 22.91. Under conventional and conservational agriculture system respectively.15.34 and 11.99 in paddy, 11.88 and 10.34 in soybean crop in conventional and conservational agriculture system respectively.

It is evident from data that in paddy crop $\left(\mathrm{C}_{1}\right)$ at initial stage $\left(\mathrm{St}_{1}\right)$ under conservational agriculture system fungi count was 4.11 percent higher than conventional agriculture system.

At reproductive stage 24.25 percent higher and at harvest13.6 pecent however the fungi count was higher in conservational agriculture system.

In maize crop $\left(\mathrm{C}_{2}\right)$ at initial stage $\left(\mathrm{St}_{1}\right)$ fungi count was 13.11 percent in reproductive stage $\left(\mathrm{St}_{2}\right) 8.74$ percent and at harvest 7.94 percent higher in conservational agriculture system. In soybean crop $\left(\mathrm{C}_{3}\right)$ at initial stage $\left(\mathrm{St}_{1}\right)$ fungi count was 9.19 percent and at reproductive stage 11.6 percent higher than conventional agriculture system. Fungi count was high in sub surface soil $\left(D_{1}\right)$ 0-15 cm than $\left(D_{2}\right) 15-30 \mathrm{~cm}$ in both the system $\left(S_{1}\right.$ and $\left.S_{2}\right)$, respectively. 
The data on interaction are presented in table 6 to evaluate the effect of crop to stage, crop and system of agriculture, the fungi population in paddy field reproductive stage $\left(\mathrm{St}_{2}\right)$ was maximum of 31.62 which was 19.15 and 19.34 at initial stage in crop maize and soybean was recorded respectively.

The interaction between depth and crop had maximum fungi count in paddy rhizosphere soil having 43.45 under depth $0-15 \mathrm{~cm} 29.38$ and 29.44 in conventional and conservational system respectively.

Under crop with depth maximum count was 0$15 \mathrm{~cm}$ in all crop having 43.45, 26.45 and 22.38 in paddy, maize and soybean rhizosphere soil. Maximum fungal count was recorded under conservational agriculture system in paddy, maize and soybean crop respectively.

\section{References}

Ajwa, H.A., Dell. CJ, and Rice.CW.1999. Changes in enzyme activities and microbial biomass of tallgrass prairie soil as related to burning and nitrogen fertilization. Journal of Soil Science Society of America Volume, May 1999, Pp. 769-777.

Frey, S.D., Elliott, ET, and Paustian.K.1999. Bacterial and fungal abundance and biomass in conventional and no-tillage agroecosystems along two climatic gradients. Soil Biology and Biochemistry, 31: $573-585$

Havlin, J.L., Kissel, DE, Maddux. LD, Claassen, MM and Long, JH. 1990. Crop Rotation and Tillage Effects on Soil
Organic Carbon and Nitrogen. Soil Science Society of America, Vol. 54 No. 2, p. 448-452.

Hridya, A.C., Byju G. 2014.Effect of chemical fertilizers and microbial inoculations on soil properties in cassava. Indian Journal of Agricultural Sciences; 84(7): 860-866.

Kulandaivelu Velmourougane, Venugopalan MV. Bhattacharyya, Dipak Sarkar; Pal, D. K., Sahu Apeksha, Chandran, P., Ray, S. K., Mandal Champa, Nair, K. M., Prasad Jagdish, Singh, R. S., Tiwari Pramod, 2014. Microbial biomass carbon status in agro-ecological sub regions of black soils in India. Journal of Biological Sciences, 84(3):519-529.

Kushwaha, C.P., Tripathi SK and Singh KP. 2000. Variations in soil microbial biomass and $\mathrm{N}$ availability due to residue and tillage management in a dryland rice agroecosystem. Soil \& Tillage Research, 56:153-166.

Martens, R., 1995. Current methods for measuring microbial biomass $\mathrm{C}$ in soil: Potentials and limitations. Review Article of BiolFertil Soils, 19:87-99.

Monrozier, L.J., Ladd. JN, Fitzpatrick. RW, Foster. RC, Rapauch M. 1991.Components and microbial biomass content of size fractions in soils of contrasting aggregation. Journal of Elsevier, Volume 50, Issues 1-2, August 1991, Pages 37-62.

Yahai Lu Akira Watanabe Makoto Kimura, 2002. Contribution of plant-derived carbon to soil microbial biomass dynamics in a paddy rice microcosm. Biol Fertil Soils, 36:136-142.

\section{How to cite this article:}

Kritika Dongre, B. Sachidanand and Porte, S.S. 2017. Assessment of Different Microbial Populationin the Rhizosphere of Main Kharif Crop under Conventional and Conservation Agriculture System. Int.J.Curr.Microbiol.App.Sci. 6(9): 813-819. doi: https://doi.org/10.20546/ijcmas.2017.609.100 